\title{
On Lie Reduction of the Navier-Stokes Equations
}

Roman POPOVYCH

Institute of Mathematics of the National Academy of Sciences of Ukraine, 3 Tereshchenkivs'ka Street, Kyiv 4, Ukraina

\begin{abstract}
Lie reduction of the Navier-Stokes equations to systems of partial differential equations in three and two independent variables and to ordinary differential equations is described.
\end{abstract}

The Navier-Stokes equations (NSEs)

$$
\vec{u}_{t}+(\vec{u} \cdot \vec{\nabla}) \vec{u}-\triangle \vec{u}+\vec{\nabla} p=\overrightarrow{0}, \quad \operatorname{div} \vec{u}=0
$$

which describe the motion of an incompressible viscous fluid are the basic equations of hydrodynamics. In (1) and below, $\vec{u}=\left\{u^{a}(t, \vec{x})\right\}$ denotes the velocity field of a fluid, $p=p(t, \vec{x})$ denotes the pressure, $\vec{x}=\left\{x_{a}\right\}, \partial_{t}=\partial / \partial t, \partial_{a}=\partial / \partial x_{a}, \vec{\nabla}=\left\{\partial_{a}\right\}, \triangle=\vec{\nabla} \cdot \vec{\nabla}$ is the Laplacian. The kinematic coefficient of viscosity and fluid density are set equal to unity.

The problem of finding exact solutions of the nonlinear equations (1) is an important and rather complicated one. There are some ways to solve it. Considerable progress in this field can be achieved by means of a symmetry approach. Equations (1) have nontrivial symmetry properties. Relatively recently, it was found by means of the Lie method $[2,1]$ that the maximal Lie invariance algebra of the NSEs (1) is the infinite-dimensional algebra $A(N S)$ with the basis elements

$$
\begin{aligned}
& D=2 t \partial_{t}+x_{a} \partial_{a}-u^{a} \partial_{u^{a}}-2 p \partial_{p}, \quad J_{a b}=x_{a} \partial_{b}-x_{b} \partial_{a}+u^{a} \partial_{u^{b}}-u^{b} \partial_{u^{a}}, a<b, \\
& \partial_{t}, \quad R(\vec{m})=R(\vec{m}(t))=m^{a} \partial_{a}+m_{t}^{a} \partial_{u^{a}}-m_{t t}^{a} x_{a} \partial_{p}, \quad Z(\chi)=Z(\chi(t))=\chi \partial_{p},
\end{aligned}
$$

where $m^{a}=m^{a}(t)$ and $\chi=\chi(t)$ are arbitrary smooth functions of $t$ (for example, from $\left.C^{\infty}\left(\left(t_{0}, t_{1}\right), \mathbf{R}\right)\right)$. Hereafter, repeated indices denote summation, whereby we consider the indices $a, b$ to take on values in $\{1,2,3\}$ and the indices $i, j$ to take on values in $\{1,2\}$. Algebra (2) contains, as a subalgebra, the eleven-dimensional extended Galilei algebra $<\partial_{t}, D, J_{a b}, \partial_{a}, G_{a}=t \partial_{a}+\partial_{u^{a}}>$.

To find exact solutions of (1), the symmetry approach in explicit form was used in a number of papers (see references in [4]). In our works $[4,7,5,6,12]$, we made a complete symmetry reduction of the NSEs to systems of PDEs in three and two independent variables and to systems of ODEs, using the subalgebraic structure of $A(N S)$.

Copyright (c) 1995 by Mathematical Ukraina Publisher. All rights of reproduction in any form reserved. 
The reduction problem for the NSEs is to describe ansatzes of the form [3]:

$$
u^{a}=f^{a b}(t, \vec{x}) v^{b}(\omega)+g^{a}(t, \vec{x}), \quad p=f^{0}(t, \vec{x}) q(\omega)+g^{0}(t, \vec{x})
$$

that reduce system (1) in four independent variables to systems of differential equations in the functions $v^{a}$ and $q$ depending on $N$ variables $\omega=\left\{\omega_{k}\right\}$, where $N$ takes on a fixed value from the set $\{1,2,3\}$. In formulas $(3), f^{a b}, g^{a}, f^{0}, g^{0}$, and $\omega_{k}$ are smooth functions to be specified. In such a general formulation, the reduction problem is too complex to solve. But using the Lie symmetry, some ansatzes (3) reducing the NSEs can be obtained.

Using the Lie method, first we constructed complete sets of $A(N S)$-inequivalent subalgebras of dimension $4-N$ for $N=3, N=2$, and $N=1$. Knowing the subalgebraic structure of $A(N S)$, we found explicit forms for the functions $f^{a b}, g^{a}, f^{0}, g^{0}$, and $\omega_{k}$ and obtained reduced systems in the functions $v^{a}$ and $q$. Due to the choice of ansatzes, the reduced systems have, in the majority of cases, rather simple forms and can be united in a smaller number of classes. We investigated symmetry properties (both classic and nonclassic) of the reduced systems of PDEs. A number of them have Lie symmetry operators that are not induced by operators from $A(N S)$. Symmetry reduction of such systems gives solutions that can not be obtained by means of direct reduction of the NSEs. We also managed to find exact solutions for most of the reduced systems of ODEs. As a result, large classes of exact solutions for the NSEs were obtained.

Let us consider the reduction of the NSEs to systems of PDEs in three independent variables. A complete set of $A(N S)$-inequivalent one-dimensional subalgebras of $A(N S)$ is exhausted by the following algebras:

1. $A_{1}^{1}(\kappa)=<D+2 \kappa J_{12}>$, where $\kappa \geq 0$.

2. $A_{2}^{1}(\kappa)=<\partial_{t}+\kappa J_{12}>$, where $\kappa \in\{0 ; 1\}$.

3. $A_{3}^{1}(\eta, \chi)=<J_{12}+R(0,0, \eta(t))+Z(\chi(t))>$ with smooth functions $\eta$ and $\chi$. Algebras $A_{3}^{1}(\eta, \chi)$ and $A_{3}^{1}(\tilde{\eta}, \tilde{\chi})$ are equivalent if $\exists \varepsilon, \delta \in \mathbf{R}, \exists \lambda \in C^{\infty}\left(\left(t_{0}, t_{1}\right), \mathbf{R}\right)$ :

$$
\tilde{\eta}(\tilde{t})=e^{-\varepsilon} \eta(t), \quad \tilde{\chi}(\tilde{t})=e^{2 \varepsilon}\left(\chi(t)+\lambda_{t t}(t) \eta(t)-\lambda(t) \eta_{t t}(t)\right),
$$

where $\tilde{t}=t e^{-2 \varepsilon}+\delta$.

4. $A_{4}^{1}(\vec{m}, \chi)=<R(\vec{m}(t))+Z(\chi(t))>$ with smooth functions $\vec{m}$ and $\chi:(\vec{m}, \chi) \not \equiv(\overrightarrow{0}, 0)$. Algebras $A_{4}^{1}(\vec{m}, \chi)$ and $A_{4}^{1}(\overrightarrow{\tilde{m}}, \tilde{\chi})$ are equivalent if $\exists \varepsilon, \delta \in \mathbf{R}, \quad \exists C \neq 0, \quad \exists B \in O(3)$, $\exists \vec{l} \in C^{\infty}\left(\left(t_{0}, t_{1}\right), \mathbf{R}^{3}\right):$

$$
\overrightarrow{\tilde{m}}(\tilde{t})=C e^{-\varepsilon} B \vec{m}(t), \quad \tilde{\chi}(\tilde{t})=C e^{2 \varepsilon}\left(\chi(t)+\vec{l}_{t t}(t) \cdot \vec{m}(t)-\vec{m}_{t t}(t) \cdot \vec{l}(t)\right),
$$

where $\tilde{t}=t e^{-2 \varepsilon}+\delta$.

By means of the algebras $A_{1}^{1}-A_{4}^{1}$ (sometimes, when additional restrictions for parameters are satisfied), we can construct ansatzes of codimension one for the Navier-Stokes field. We list these ansatzes and the corresponding reduced systems.

Ansatzes constructed by means of the algebras $A_{1}^{1}$ and $A_{2}^{1}$ have the following forms:

1. $u^{1}=|t|^{-1 / 2}\left(v^{1} \cos \tau-v^{2} \sin \tau\right)+\frac{1}{2} x_{1} t^{-1}-\kappa x_{2} t^{-1}$,

$u^{2}=|t|^{-1 / 2}\left(v^{1} \sin \tau+v^{2} \cos \tau\right)+\frac{1}{2} x_{2} t^{-1}+\kappa x_{1} t^{-1}$,

$u^{3}=|t|^{-1 / 2} v^{3}+\frac{1}{2} x_{3} t^{-1}$,

$p=|t|^{-1} q+\frac{1}{2} \kappa^{2} t^{-2} r^{2}+\frac{1}{8} t^{-2} x_{a} x_{a}$, 
where $y_{1}=|t|^{-1 / 2}\left(x_{1} \cos \tau+x_{2} \sin \tau\right), y_{2}=|t|^{-1 / 2}\left(-x_{1} \sin \tau+x_{2} \cos \tau\right), y_{3}=|t|^{-1 / 2} x_{3}$, $\tau=\kappa \ln |t|$. Here and below, $v^{a}=v^{a}\left(y_{1}, y_{2}, y_{3}\right), q=q\left(y_{1}, y_{2}, y_{3}\right), r=\left(x_{1}^{2}+x_{2}^{2}\right)^{1 / 2}$.

2. $u^{1}=v^{1} \cos \kappa t-v^{2} \sin \kappa t-\kappa x_{2}$,

$u^{2}=v^{1} \sin \kappa t+v^{2} \cos \kappa t+\kappa x_{1}$,

$u^{3}=v^{3}$,

$p=q+\frac{1}{2} \kappa^{2} r^{2}$,

where

$y_{1}=x_{1} \cos \kappa t+x_{2} \sin \kappa t, \quad y_{2}=-x_{1} \sin \kappa t+x_{2} \cos \kappa t, \quad y_{3}=x_{3}$.

Substituting ansatzes (6) and (7) into the NSEs, we obtain reduced systems of PDEs with the same general form

$$
\begin{aligned}
& v^{a} v_{a}^{1}-v_{a a}^{1}+q_{1}+\gamma_{1} v^{2}=0, \\
& v^{a} v_{a}^{2}-v_{a a}^{2}+q_{2}-\gamma_{1} v^{1}=0, \\
& v^{a} v_{a}^{3}-v_{a a}^{3}+q_{3}=0, \\
& v_{a}^{a}=\gamma_{2}
\end{aligned}
$$

where $\gamma_{1}=-2 \kappa \operatorname{sign} t, \gamma_{2}=-\frac{3}{2} \operatorname{sign} t$ for ansatz (6) and $\gamma_{1}=-2 \kappa, \gamma_{2}=0$ for ansatz (7). Hereafter, subscripts 1,2 , and 3 of functions denote differentiation with respect to $y_{1}, y_{2}$, and $y_{3}$, accordingly.

All Lie symmetry operators of system (9) are induced by operators from $A(N S)$.

We should like to emphasize again that selection of a "good" form for an ansatz is important for reduction to be successful. Our problem is to find or "to guess", at once, such an ansatz that the corresponding reduced system have a simple and convenient form for our investigation. Moreover, selection of an ansatz is needed for Lie symmetry operators of the reduced system to have simple and "natural" forms too. The ansatzes constructed by us satisfy these demands. Let us illustrate this statement by the simple example of the algebra $A_{2}^{1}$.

There exists an infinite set of choices for the invariant independent variables $y_{a}$. For example, we can take the following expressions for them:

$$
y_{1}=\arctan \frac{x_{2}}{x_{1}}-\kappa t, \quad y_{2}=\left(x_{1}^{2}+x_{2}^{2}\right)^{1 / 2}, \quad y_{3}=x_{3} .
$$

However choosing $y_{a}$ in such a way, we obtain a reduced system which strongly differs from the "natural" reduced system for $\kappa=0$ (i.e., the NSEs for steady flows of a viscous fluid in Cartesian coordinates). It is better to choose the variables $y_{a}$ in form (8).

To construct an ansatz, the functions $f^{a b}, f^{0}, g^{a}$, and $g^{0}$ are to be found too. We can take, for example, such values of these functions which give an ansatz of the following simple form:

$$
u^{1}=\tilde{v}^{1} \cos \kappa t-\tilde{v}^{2} \sin \kappa t, \quad u^{2}=\tilde{v}^{1} \sin \kappa t+\tilde{v}^{2} \cos \kappa t, \quad u^{3}=\tilde{v}^{3}, \quad p=\tilde{q},
$$

where $\tilde{v}^{a}=\tilde{v}^{a}\left(y_{1}, y_{2}, y_{3}\right)$ and $\tilde{q}=\tilde{q}\left(y_{1}, y_{2}, y_{3}\right)$. Substituting ansatz (10) into the NSEs, we obtain the reduced system on the new unknown functions $\tilde{v}^{a}$ and $\tilde{q}$, having variable 
coefficients. It can be simplified to system (9), having constant coefficients, by means of the local transformation

$$
\tilde{v}^{1}=v^{1}-\kappa y_{2}, \quad \tilde{v}^{2}=v^{2}+\kappa y_{1}, \quad \tilde{v}^{3}=v^{3}, \quad \tilde{q}=q+\frac{1}{2}\left(y_{1}^{2}+y_{2}^{2}\right) .
$$

But simpler ansatz (10) is transformed under (11) into more complicated ansatz (7). Therefore, we can take ansatz (7) and obtain system (9) at once.

The above is a good example how a reduced system can be simplified by means of modifying (complicating) an ansatz corresponding to it.

An ansatz constructed by means of the algebra $A_{3}^{1}(\eta, \chi)$ has the following form:

3. $u^{1}=x_{1} r^{-1} v^{1}-x_{2} r^{-1} v^{2}+x_{1} r^{-2}$,

$$
\begin{aligned}
& u^{2}=x_{2} r^{-1} v^{1}+x_{1} r^{-1} v^{2}+x_{2} r^{-2}, \\
& u^{3}=v^{3}+\eta(t) r^{-1} v^{2}+\eta_{t}(t) \arctan x_{2} / x_{1}, \\
& p=q-\frac{1}{2} \eta_{t t}(t)(\eta(t))^{-1} x_{3}^{2}-\frac{1}{2} r^{-2}+\chi(t) \arctan x_{2} / x_{1},
\end{aligned}
$$

where $\quad y_{1}=t, \quad y_{2}=r, \quad y_{3}=x_{3}-\eta(t) \arctan x_{2} / x_{1}$.

The corresponding reduced system is very complicated and all its Lie symmetry operators are induced by operators from $A(N S)$ too. When $\eta=\chi=0$, this system describes axially symmetric motion of a fluid and can be transformed into a system of two equations for a stream function $\Psi^{1}$ and a function $\Psi^{2}$ that are determined by the equations

$$
\Psi_{3}^{1}=y_{2} v^{1}, \quad \Psi_{2}^{1}=-y_{2} v^{3}, \quad \Psi^{2}=y_{2} v^{2} .
$$

The transformed system was studied by L.V. Kapitanskiy [9, 10]. He also described techniques for classifying inequivalent subalgebras of infinite-dimensional algebras of form (2).

Ansatz corresponding to the algebra $A_{4}^{1}(\vec{m}, \chi)$ can be constructed only for such $t$ that $\vec{m}(t) \neq \overrightarrow{0}$. For these values of $t$, the parameter-function $\chi$ can be made to vanish by means of equivalence transformation (5). An ansatz constructed with the algebra $A_{4}^{1}(\vec{m}, 0)$ and the corresponding reduced system have the following forms

4. $\quad \vec{u}=v^{i} \vec{n}^{i}+|\vec{m}|^{-2} v^{3} \vec{m}+|\vec{m}|^{-2}(\vec{m} \cdot \vec{x}) \vec{m}_{t}-y_{i} \vec{n}_{t}^{i}$,

$$
\begin{aligned}
p= & q-\frac{3}{2}|\vec{m}|^{-2}\left(\left(\vec{m}_{t} \cdot \vec{n}^{i}\right) y_{i}\right)^{2}-|\vec{m}|^{-2}\left(\vec{m}_{t t} \cdot \vec{x}\right)(\vec{m} \cdot \vec{x})+ \\
& +\frac{1}{2}\left(\vec{m}_{t t} \cdot \vec{m}\right)|\vec{m}|^{-4}(\vec{m} \cdot \vec{x})^{2},
\end{aligned}
$$

where $\quad y_{i}=\vec{n}^{i} \cdot \vec{x}, \quad y_{3}=t, \quad \vec{n}^{i} \cdot \vec{m}=\vec{n}^{1} \cdot \vec{n}^{2}=\vec{n}_{t}^{1} \cdot \vec{n}^{2}=0, \quad\left|\vec{n}^{i}\right|=1 \quad$ and

4. $v_{3}^{i}+v^{j} v_{j}^{i}-v_{j j}^{i}+q_{i}+\rho^{i} v^{3}=0$,

$$
\begin{aligned}
& v_{3}^{3}+v^{j} v_{j}^{3}-v_{j j}^{3}=0, \\
& v_{i}^{i}+\rho^{3}=0,
\end{aligned}
$$

where $\rho^{i}=\rho^{i}\left(y_{3}\right)=2|\vec{m}|^{-2}\left(\vec{m}_{t} \cdot \vec{n}^{i}\right), \quad \rho^{3}=\rho^{3}\left(y_{3}\right)=|\vec{m}|^{-2}\left(\vec{m}_{t} \cdot \vec{m}\right)$. For arbitrary values of the functions $\rho^{a}$, system (13) is invariant under the operators

$$
R_{3}\left(\psi^{1}(t), \psi^{2}(t)\right)=\psi^{i} \partial_{y_{i}}+\psi_{t}^{i} \partial_{v^{i}}-\psi_{t t}^{i} y_{i} \partial_{q}, \quad Z^{1}(\lambda(t))=\lambda \partial_{q}, \quad S=\partial_{v^{3}}-\rho^{i} y_{i} \partial_{q}
$$


which are induced by operators from $A(N S)$. But for some values of $\rho^{a}$, system (13) additionally admits Lie symmetry operators contained in the span of the operators

$$
\begin{aligned}
& E(\chi(t))=2 \chi \partial_{t}+\chi_{t} y_{i} \partial_{y_{i}}+\left(\chi_{t t} y_{i}-\chi_{t} v^{i}\right) \partial_{v^{i}}-\left(2 \chi_{t} q+\frac{1}{2} \chi_{t t t} y_{j} y_{j}\right) \partial_{q}, \\
& J_{12}^{1}=y_{1} \partial_{2}-y_{2} \partial_{1}+v^{1} \partial_{v^{2}}-v^{2} \partial_{v^{1}}, \quad I=v^{3} \partial_{v^{3}} .
\end{aligned}
$$

For example, if $\rho^{i}=0$, the maximal Lie invariance algebra of (13) is the algebra

$$
<R_{3}\left(\psi^{1}(t), \psi^{2}(t)\right), Z^{1}(\lambda(t)), S, J_{12}^{1}, E\left(\chi^{1}(t)\right), E\left(\chi^{2}(t)\right), I>,
$$

where $\chi^{1}=e^{-\rho(t)} \int e^{\rho(t)} d t, \chi^{2}=e^{-\rho(t)}, \rho=\int \rho^{3}(t) d t, t=y_{3}$. The operators $E\left(\chi^{i}\right)$ and $I$ are not induced by operators from $A(N S)$ if $\rho^{3}(t) \neq \alpha_{1}\left(\alpha_{2} t+\alpha_{3}\right)^{-1}$, where $\alpha_{b}=$ const.

We reduced system (13) by means of the algebras containing the "nontrivial" operators $E\left(\chi^{i}\right)$ and $I$ to systems in one and two independent variables and constructed their exact solutions. Investigation of system (13) is simplified due to the function $\rho^{3}$ can be made to vanish by means of a local transformation. It is interesting to note that a number of the reduced systems are analogous to ones obtained from the NSEs directly. And we can investigate them only once. This situation is reiterated.

Let us proceed to the reduction of the NSEs to systems in two independent variables. A complete set of $A(N S)$-inequivalent two-dimensional subalgebras is exhausted by twelve algebras. By means of eight of them, we can constructed ansatzes that reduce the NSEs to five classes of systems. Two reduced systems are very complicated and all their Lie symmetry operators are induced by operators from $A(N S)$. But the other systems are very interesting.

So, an ansatz constructed with the algebra $A_{1}^{2}\left(\vec{m}^{1}, \vec{m}^{2}\right)=<R\left(\vec{m}^{1}(t)\right), R\left(\vec{m}^{2}(t)\right)>$, where $\vec{m}_{t t}^{1} \cdot \vec{m}^{2}-\vec{m}^{1} \cdot \vec{m}_{t t}^{2}=0$ and $\operatorname{rank}\left(\vec{m}^{1}, \vec{m}^{2}\right)=2$, has the form

1. $\vec{u}=\vec{w}+\lambda^{-1}\left(\vec{n}^{i} \cdot \vec{x}\right) \vec{m}_{t}^{i}-\lambda^{-1}(\vec{k} \cdot \vec{x}) \vec{k}_{t}$,

$$
p=s-\frac{1}{2} \lambda^{-1}\left(\vec{m}_{t t}^{i} \cdot \vec{x}\right)\left(\vec{n}^{i} \cdot \vec{x}\right)-\frac{1}{2} \lambda^{-2}\left(m_{t t}^{i} \cdot \vec{k}\right)\left(\vec{n}^{i} \cdot \vec{x}\right)(\vec{k} \cdot \vec{x}),
$$

where $z_{1}=t, \quad z_{2}=(\vec{k} \cdot \vec{x}), \vec{k}=\vec{m}^{1} \times \vec{m}^{2}, \vec{n}^{1}=\vec{m}^{2} \times \vec{k}, \vec{n}^{2}=\vec{k} \times \vec{m}^{1}, \quad \lambda=\lambda(t)=\vec{k} \cdot \vec{k} \neq 0$ $\forall t \in\left(t_{0}, t_{1}\right)$. Here and below $\vec{w}=\vec{w}\left(z_{1}, z_{2}\right), s=s\left(z_{1}, z_{2}\right)$.

Substituting ansatz (14) into the NSEs, we obtain the following reduced system:

1. $\vec{w}_{1}-\lambda \vec{w}_{22}+s_{2} \vec{k}+(\vec{k} \cdot \vec{w})\left(\vec{w}-\lambda^{-1} \vec{k}_{t}\right)+\lambda^{-1}\left(\vec{n}^{i} \cdot \vec{w}\right) \vec{m}_{t}^{i}+z_{2} \vec{e}=\overrightarrow{0}$,

$$
\vec{k} \cdot \vec{w}_{2}=0,
$$

where $\vec{e}=\vec{e}(t)=2 \lambda^{-2}\left(\vec{m}_{t}^{1} \cdot \vec{m}^{2}-\vec{m}^{1} \cdot \vec{m}_{t}^{2}\right) \vec{k}_{t} \times \vec{k}+\lambda^{-2}\left(2 \vec{k}_{t} \cdot \vec{k}_{t}-\vec{k}_{t t} \cdot \vec{k}\right)$ and $t=z_{1}$.

The general solution (15) is expressed in terms of the general solution of the decomposed system of two linear one-dimensional heat equations. As a result, we obtain two classes of solutions for the NSEs:

Case A. $\vec{m}_{t}^{1} \cdot \vec{m}^{2}-\vec{m}^{1} \cdot \vec{m}_{t}^{2}=0$ :

$$
\begin{aligned}
\vec{u}= & \lambda^{-1}\left(g_{\zeta}^{i}(\tau, \zeta)+\vec{m}_{t}^{i} \cdot \vec{x}\right) \vec{n}^{i}-\lambda^{-1}\left(\vec{k}_{t} \cdot \vec{x}\right) k, \\
p= & 2 \lambda^{-2}\left(\vec{n}^{i} \cdot \vec{k}_{t}\right) g^{i}(\tau, \zeta)+ \\
& +\frac{1}{2} \lambda^{-2}\left(\vec{k}_{t t} \cdot \vec{k}-2 \vec{k}_{t} \cdot \vec{k}_{t}\right) \zeta^{2}-\frac{1}{2} \lambda^{-1}\left(\vec{n}^{i} \cdot \vec{x}\right)\left(\vec{m}_{t t}^{i} \cdot \vec{x}\right)-\frac{1}{2} \lambda^{-2}\left(\vec{k} \cdot \vec{m}_{t t}^{i}\right)\left(\vec{n}^{i} \cdot \vec{x}\right) \zeta,
\end{aligned}
$$


Case B. $\vec{m}_{t}^{1} \cdot \vec{m}^{2}-\vec{m}^{1} \cdot \vec{m}_{t}^{2}=1$ :

$$
\begin{aligned}
\vec{u}= & \lambda^{-1}\left(\theta^{i j}(t) g_{\zeta}^{j}(\tau, \zeta)+\theta^{i 0}(t) \zeta+\vec{m}_{t}^{i} \cdot \vec{x}-\lambda^{-1}\left(\left(\vec{k} \times \vec{m}^{i}\right) \cdot \vec{x}\right)\right) \vec{n}^{i}-\lambda^{-1}(\vec{k} t \cdot \vec{x}) \vec{k}, \\
p= & 2 \lambda^{-2}\left(\vec{n}^{i} \cdot \vec{k}_{t}\right)\left(\theta^{i j}(t) g^{j}(\tau, \zeta)+\frac{1}{2} \theta^{i 0}(t) \zeta^{2}\right)+ \\
& +\frac{1}{2} \lambda^{-2}\left(\vec{k}_{t t} \cdot \vec{k}-2 \vec{k}_{t} \cdot \vec{k}_{t}\right) \zeta^{2}-\frac{1}{2} \lambda^{-1}\left(\vec{n}^{i} \cdot \vec{x}\right)\left(\vec{m}_{t t}^{i} \cdot \vec{x}\right)-\frac{1}{2} \lambda^{-2}\left(\vec{k} \cdot \vec{m}_{t t}^{i}\right)\left(\vec{n}^{i} \cdot \vec{x}\right) \zeta .
\end{aligned}
$$

Here $\zeta=\vec{k} \cdot \vec{x}, \quad \tau=\int \lambda(t) d t$, and

$$
g_{\tau}^{1}-g_{\zeta \zeta}^{1}=0, \quad g_{\tau}^{2}-g_{\zeta \zeta}^{2}=0 .
$$

$\left(\theta^{1 i}(t), \theta^{2 i}(t)\right)(i=1,2)$ are linearly independent solutions of the system

$$
\theta_{t}^{i}+\lambda^{-1}\left(\vec{m}^{i} \cdot \vec{m}^{2}\right) \theta^{1}-\lambda^{-1}\left(\vec{m}^{i} \cdot \vec{m}^{1}\right) \theta^{2}=0,
$$

and $\left(\theta^{10}(t), \theta^{20}(t)\right)$ is a particular solution of the nonhomogeneous system

$$
\theta_{t}^{i}+\lambda^{-1}\left(\vec{m}^{i} \cdot \vec{m}^{2}\right) \theta^{1}-\lambda^{-1}\left(\vec{m}^{i} \cdot \vec{m}^{1}\right) \theta^{2}=2 \lambda^{-2}\left(\left(\vec{k} \times \vec{k}_{t}\right) \cdot \vec{m}^{i}\right) .
$$

Both classes contain two arbitrary solutions of the heat equation and five arbitrary functions of $t$. The Lie symmetry of the linear one-dimensional heat equations is well known. It principally differs from one of the NSEs. The Q-conditional symmetry of the heat equation was investigated in [8] completely. Moreover, being decomposed, system (16) admits transformations where the independent variables can be transformed in the functions $g^{1}$ and $g^{2}$ in different ways. Such transformations are non-Lie ones. Large sets of exact solutions for the heat equations are known too.

In an analogous way, an ansatz constructed with the algebra $A_{2}^{2}(\lambda, \rho)=<J_{12}+$ $R(0,0, \lambda)+Z\left(\chi_{t}(t)\right), R(0,0, \rho)>$, where $\rho \neq \equiv 0$ and $\lambda_{t} \rho-\lambda \rho_{t}=: \varepsilon \in\{0 ; 1\}$, has the form

2. $u^{1}=x_{1} w^{1}-x_{2} r^{-2}\left(w^{2}-\chi(t)\right)$,

$u^{2}=x_{2} w^{1}+x_{1} r^{-2}\left(w^{2}-\chi(t)\right)$,

$u^{3}=(\rho(t))^{-1}\left(w^{3}+\rho_{t}(t) x_{3}+\varepsilon \arctan x_{2} / x_{1}\right)$,

$p=s-\frac{1}{2} \rho_{t t}(t)(\rho(t))^{-1} x_{3}^{2}+\chi_{t}(t) \arctan x_{2} / x_{1}$,

where $z_{1}=t, z_{2}=r=\left(x_{1}^{2}+x_{2}^{2}\right)^{1 / 2}$.

Substituting ansatz (19) into the NSEs, we obtain the following reduced system:

2. $w_{1}^{1}+\left(w^{1}\right)^{2}-z_{2}^{-4}\left(w^{2}-\chi\right)^{2}+z_{2} w^{1} w_{2}^{1}-w_{22}^{1}-3 z_{2} w_{2}^{1}+z_{2}^{-1} s_{2}=0$,

$w_{1}^{2}+z_{2} w^{1} w_{2}^{2}-w_{22}^{2}+z_{2}^{-1} w_{2}^{2}=0$

$w_{1}^{3}+z_{2} w^{1} w_{2}^{3}-w_{22}^{3}-z_{2}^{-1} w_{2}^{3}+z_{2}^{-2}\left(w^{2}-\chi\right)=0$,

$2 w^{1}+z_{2} w_{2}^{1}+\rho_{1} / \rho=0$.

System (20) implies the following expressions for the functions $w^{3}$ and $q$ :

$$
\begin{aligned}
& w^{1}=-\frac{1}{2} \rho_{t} \rho^{-1}+(\eta-1) z_{2}^{-2}, \\
& q=\frac{1}{4}\left(\left(\rho_{t} \rho^{-1}\right)_{t}-\frac{1}{2}\left(\rho_{t} \rho^{-1}\right)^{2}\right) z_{2}^{2}-\eta_{t} \ln \left|z_{2}\right|-\frac{1}{2}(\eta-1)^{2} z_{2}^{-2}+\int\left(w^{2}-\chi\right)^{2} z_{2}^{-3} d z_{2},
\end{aligned}
$$


and the following linear system in the functions $w^{i}$ :

$$
w_{\tau}^{2}-w_{\zeta \zeta}^{2}+\eta \zeta^{-1} w_{\zeta}^{2}=0, \quad w_{\tau}^{3}-w_{\zeta \zeta}^{3}+(\eta-2) \zeta^{-1} w_{\zeta}^{3}+\varepsilon\left(w^{2}-\chi\right) \zeta^{-2}=0
$$

where $\tau=\int|\rho(t)| d t, \quad \zeta=|\rho(t)|^{1 / 2} z_{2}$, and $\eta=\eta(t)$ is an arbitrary smooth function of $z_{1}=t$.

If $\varepsilon=0$, system (21) is decomposed and consists of two translational linear equations of the same general form [13]

$$
f_{\tau}+\eta(\tau) \zeta^{-1} f_{\zeta}-f_{\zeta \zeta}=0
$$

When $\eta$ vanishes, equation (22) is the heat equation, and, when $\eta=-2$, it is reduced to the heat equation by means of the local transformation $\tilde{f}=\zeta f$. For the other constant values of $\eta$, the maximal Lie invariance algebra of (22) is generated by the operators

$$
\begin{aligned}
& \partial_{\tau}, \quad \hat{D}=2 \tau \partial_{\tau}+\zeta \partial_{\zeta}, \quad \hat{I}=f \partial_{f}, \\
& \Pi=4 \tau^{2} \partial_{\tau}+4 \tau \zeta \partial_{\zeta}-\left(\zeta^{2}+2(1-\eta) \tau\right) f \partial_{f}, \quad g(\tau, \zeta) \partial_{f},
\end{aligned}
$$

where $g=g(\tau, \zeta)$ is an arbitrary solution of $(22)$. The operator $\Pi$ principally differs from the symmetry operators of the NSEs. Full Lie reduction of (22) with $\eta=$ const was made. The equations for the coefficients of Q-conditional symmetry operators of (22) were obtained in the general case. It was shown that, by means of nonlocal transformations, these equations are reduced to (22). Some Q-conditional symmetry operators, for example,

$$
\partial_{\zeta}, \quad X=\partial_{\tau}+(\eta-1) \zeta^{-1} \partial_{\zeta}, \quad G=(2 \tau+C) \partial_{\zeta}-\zeta f \partial_{f} \quad(C=\text { const })
$$

were found in explicit forms. Using them, large classes of non-Lie solutions of (22) were constructed. A nonlocal invariance transformation for the class of equations (22) was found.

System (21) with $\varepsilon=1$ was investigated in the same way.

The last interesting case of reduction to systems in two independent variables is given by four algebras:

3. $A_{3}^{2}(\sigma, \kappa, \mu, \nu, \varepsilon)=<D+2 \kappa J_{12}, R\left(|t|^{\sigma+1 / 2}(\nu \cos \tau, \nu \sin \tau, \mu)\right)+Z\left(\varepsilon|t|^{\sigma-1}\right)>$, where $\tau=\kappa \ln |t|, \kappa>0, \mu \geq 0, \nu \geq 0, \mu^{2}+\nu^{2}=1, \varepsilon \sigma=0$, and $\varepsilon \geq 0$.

4. $A_{4}^{2}(\sigma, \varepsilon)=<D, R\left(0,0,|t|^{\sigma+1 / 2}\right)+Z\left(\varepsilon|t|^{\sigma-1}\right)>$, where $\varepsilon \sigma=0$ and $\varepsilon \geq 0$.

5. $A_{5}^{2}(\sigma, \mu, \nu, \varepsilon)=<\partial_{t}+J_{12}, R\left(\nu e^{\sigma t} \cos t, \nu e^{\sigma t} \sin t, \mu e^{\sigma t}\right)+Z\left(\varepsilon e^{\sigma t}\right)>$, where $\mu \geq 0$, $\nu \geq 0, \mu^{2}+\nu^{2}=1, \varepsilon \sigma=0$, and $\varepsilon \geq 0$.

6. $A_{6}^{2}(\sigma, \varepsilon)=<\partial_{t}, R\left(0,0, e^{\sigma t}\right)+Z\left(\varepsilon e^{\sigma t}\right)>$, where $\sigma \in\{-1 ; 0 ; 1\}, \varepsilon \sigma=0$, and $\varepsilon \geq 0$.

Ansatzes constructed by means of the algebras $A_{3}^{2}-A_{6}^{2}$ are very cumbersome but have 
simple and similar structure:

3. $u^{1}=|t|^{-1 / 2}\left(\mu w^{1}+\nu w^{3}\right) \cos \tau-|t|^{-1 / 2} w^{2} \sin \tau+$

$$
\begin{aligned}
& +\nu \xi t^{-1} \cos \tau+\frac{1}{2} t^{-1} x_{1}-\kappa t^{-1} x_{2}, \\
u^{2}= & |t|^{-1 / 2}\left(\mu w^{1}+\nu w^{3}\right) \sin \tau+|t|^{-1 / 2} w^{2} \cos \tau+ \\
& +\nu \xi t^{-1} \sin \tau+\frac{1}{2} t^{-1} x_{2}+\kappa t^{-1} x_{1}, \\
u^{3}= & |t|^{-1 / 2}\left(-\nu w^{1}+\mu w^{3}\right)+\mu \xi t^{-1}+\frac{1}{2} t^{-1} x_{3}, \\
p= & |t|^{-1} s-\frac{1}{2} t^{-2} \xi^{2}+\frac{1}{8} t^{-2} R^{2}+\frac{1}{2} \kappa^{2} t^{-2} r^{2}+ \\
& +\varepsilon|t|^{-3 / 2}\left(\nu x_{1} \cos \tau+\nu x_{2} \sin \tau+\mu x_{3}\right),
\end{aligned}
$$

where

$$
\begin{aligned}
& z_{1}=|t|^{-1 / 2}\left(\mu x_{1} \cos \tau+\mu x_{2} \sin \tau-\nu x_{3}\right), \\
& z_{2}=|t|^{-1 / 2}\left(x_{2} \cos \tau-x_{1} \sin \tau\right), \\
& \xi=\sigma\left(\nu x_{1} \cos \tau+\nu x_{2} \sin \tau+\mu x_{3}\right)+2 \kappa \nu\left(x_{2} \cos \tau-x_{1} \sin \tau\right), \quad \tau=\kappa \ln |t| .
\end{aligned}
$$

4. $u^{1}=|t|^{-1 / 2} w^{1}+\frac{1}{2} t^{-1} x_{1}$,

$u^{2}=|t|^{-1 / 2} w^{2}+\frac{1}{2} t^{-1} x_{2}$,

$u^{3}=|t|^{-1 / 2} w^{3}+\left(\sigma+\frac{1}{2}\right) t^{-1} x_{3}$,

$p=|t|^{-1} s-\frac{1}{2} \sigma^{2} t^{-2} x_{3}^{2}+\frac{1}{8} t^{-2} R^{2}+\varepsilon|t|^{-3 / 2} x_{3}$,

where $\quad z_{1}=|t|^{-1 / 2} x_{1}, \quad z_{2}=|t|^{-1 / 2} x_{2}$.

5. $u^{1}=\left(\mu w^{1}+\nu w^{3}\right) \cos t-w^{2} \sin t+\nu \xi \cos t-x_{2}$,

$u^{2}=\left(\mu w^{1}+\nu w^{3}\right) \sin t+w^{2} \cos t+\nu \xi \sin t+x_{1}$,

$u^{3}=\left(-\nu w^{1}+\mu w^{3}\right)+\mu \xi$,

$p=s-\frac{1}{2} \xi^{2}+\frac{1}{2} r^{2}+\varepsilon\left(\nu x_{1} \cos t+\nu x_{2} \sin t+\mu x_{3}\right)$,

where

$z_{1}=\left(\mu x_{1} \cos t+\mu x_{2} \sin t-\nu x_{3}\right), \quad z_{2}=\left(x_{2} \cos t-x_{1} \sin t\right)$,

$\xi=\sigma\left(\nu x_{1} \cos t+\nu x_{2} \sin t+\mu x_{3}\right)+2 \nu\left(x_{2} \cos t-x_{1} \sin t\right)$,

6. $u^{1}=w^{1}, \quad u^{2}=w^{2}, \quad u^{3}=w^{3}+\sigma x_{3}, \quad p=s-\frac{1}{2} \sigma^{2} x_{3}^{2}+\varepsilon x_{3}$,

where $z_{1}=x_{1}, \quad z_{2}=x_{2}$.

Ansatzes (23)-(26) reduce the NSEs to the system having the following simple general form:

$$
\begin{aligned}
& w^{i} w_{i}^{1}-w_{i i}^{1}+s_{1}+\alpha_{2} w^{2}=0, \\
& w^{i} w_{i}^{2}-w_{i i}^{2}+s_{2}-\alpha_{2} w^{1}+\alpha_{1} w^{3}=0, \\
& w^{i} w_{i}^{3}-w_{i i}^{3}+\alpha_{4} w^{3}+\alpha_{5}=0 \\
& w_{i}^{i}=\alpha_{3}
\end{aligned}
$$


where the constants $\alpha_{n}(n=\overline{1,5})$ take on the values
4. $\alpha_{1}= \pm 2 \kappa \nu$,
$\alpha_{2}=\mp 2 \kappa \mu$,
$\alpha_{3}=\mp(\sigma+3 / 2)$,
$\alpha_{4}= \pm \sigma, \quad \alpha_{5}=\varepsilon$.
5. $\alpha_{1}=0$,
$\alpha_{2}=0$,
$\alpha_{3}=\mp(\sigma+3 / 2)$,
$\alpha_{4}= \pm \sigma, \quad \alpha_{5}=\varepsilon$.
6. $\alpha_{1}=2 \nu$,
$\alpha_{2}=-2 \mu$,
$\alpha_{3}=-\sigma$,
$\alpha_{4}=\sigma, \quad \alpha_{5}=\varepsilon$.
7. $\alpha_{1}=0$,
$\alpha_{2}=0$,
$\alpha_{3}=-\sigma$,
$\alpha_{4}=\sigma, \quad \alpha_{5}=\varepsilon$.

System (27) was known for some values of $\alpha_{n}$. Setting $\alpha_{k}=0(k=\overline{2,5})$ in $(27)$, we obtain equations describing a plane convective flow that is brought about by nonhomogeneous heating of boudaries. In this case, $w^{i}$ are the coordinates of the flow velocity vector, $w^{3}$ is the flow temperature, $s$ is the pressure, the Grasshoff number $\lambda$ is equal to $-\alpha_{1}$, and the Prandtl number $\sigma$ is equal to 1. Some similarity solutions of these equations were constructed in [11]. The particular case of system (27) for $\alpha_{1}=\alpha_{2}=\alpha_{4}=\alpha_{5}=0$ and $\alpha_{3}=1$ was considered in [14]. Moreover, system (27) is obtained as a result of nondirect reduction of the NSEs by means of noninduced operators too. That is why, we investigated this system in detail.

For arbitrary values of the $\alpha_{n}$, system (27) is invariant under the operators $\partial_{1}, \partial_{2}, \partial_{s}$. There are nine cases of extending the maximal Lie invariance algebra of (27) by operators from the span $<\partial_{w^{3}}-\alpha_{1} z_{2} \partial_{s}, z_{i} \partial_{i}-w^{i} \partial_{w^{i}}-2 s \partial_{s}, z_{1} \partial_{2}-z_{2} \partial_{1}+w^{1} \partial_{w^{2}}-w^{2} \partial_{w^{1}}, w^{3} \partial_{w^{3}}>$. If $\alpha_{1}=\alpha_{4}=\alpha_{5}=0$, the maximal Lie invariance algebra of (27) contains the operator $w^{3} \partial_{w^{3}}$ that is not induced by elements from $A(N S)$.

Making the nonlocal transformation

$$
\tilde{s}=s+\alpha_{2} \Psi,
$$

where $\Psi_{1}=w^{2}, \Psi_{2}=-w^{1}$ (such a function $\Psi$ exists in view of the last equation of (27)), in system (27) with $\alpha_{3}=0$, we obtain a system of the form (27) with $\tilde{\alpha}_{3}=\tilde{\alpha}_{2}=0$. In some cases $\left(\alpha_{1} \neq 0, \alpha_{3}=\alpha_{4}=\alpha_{5}=0, \alpha_{2} \neq 0 ; \alpha_{1}=\alpha_{3}=\alpha_{4}=0, \alpha_{2} \neq 0\right)$, transformation (28) allows the symmetry of (27) to be extended and non-Lie solutions to be constructed. Moreover, it means that in the cases listed above, system (27) is invariant under the nonlocal transformation

$$
\hat{z}_{i}=e^{\varepsilon} z_{i}, \quad \hat{w}^{i}=e^{-\varepsilon} w^{i}, \quad \hat{w}^{3}=e^{\delta \varepsilon} w^{3}, \quad \hat{s}=e^{-2 \varepsilon} s+\alpha_{2}\left(e^{-2 \varepsilon}-1\right) \Psi,
$$

where the parameter $\delta$ takes on a fixed value from the set $\{-3 ; 0 ; 2\}$, depending on the values of $\alpha_{n}$.

To reduce system (27) to ODEs, we used the following ansatz:

$$
\begin{aligned}
w^{1} & =a_{1} \varphi^{1}-a_{2} \varphi^{3}+b_{1} \zeta, \\
w^{2} & =a_{2} \varphi^{1}+a_{1} \varphi^{3}+b_{2} \zeta, \\
w^{3} & =\varphi^{2}+b_{3} \zeta, \\
s & =h+d_{1} \zeta+d_{2} \omega \zeta+\frac{1}{2} d_{3} \zeta^{2},
\end{aligned}
$$

where $\omega=a_{1} z_{2}-a_{2} z_{1}, \quad \zeta=a_{1} z_{1}+a_{2} z_{2}, \quad \varphi^{a}=\varphi^{a}(\omega), \quad h=h(\omega), \quad a_{i}, b_{a}, d_{a}, B=$ const, $a_{1}^{2}+a_{2}^{2}=1$,

$$
b_{i}=B a_{i}, \quad b_{3}\left(B+\alpha_{4}\right)=0, \quad d_{2}=\alpha_{2} B-\alpha_{1} b_{3} a_{1}, \quad d_{3}=-B^{2}-\alpha_{1} b_{3} a_{2} .
$$


Indeed, formulae (29) and (30) determine a whole set of ansatzes for system (27). This set contains both Lie ansatzes, constructed by means of subalgebras of the form

$$
<a_{1} \partial_{1}+a_{2} \partial_{2}+a_{3}\left(\partial_{w^{3}}-\alpha_{1} z_{2} \partial_{s}\right)+a_{4} \partial_{s}>,
$$

and non-Lie ansatzes. Equation (30) is the necessary and sufficient condition to reduce (27) by means of an ansatz of form (28).

Full Lie reduction of system (27) to ODEs was made by means of the subalgebras that do not belong to the set of algebras (31).

Let us describe reduction of the NSEs to systems of ODEs briefly. A complete set of $A(N S)$-inequivalent three-dimensional subalgebras of $A(N S)$ is exhausted by 52 classes of algebras. By means of 22 "good" classes from this set, one can construct ansatzes of codimension three for the Navier-Stokes field. We united the "good" classes to 8 superclasses. By means of them, we constructed 8 ansatzes and obtained reduced systems which can be united to 6 classes. Either general or particular solutions for the reduced systems were obtained. 


\section{References}

[1] Bytev V.O., Group properties of the Navier-Stokes equations, in Chislennye metody mehaniki sploshnoy sredy, V.3, 13-17, Comp. center Siberian Dep. Acad. Sci. USSR, Novosibirsk, 1972.

[2] Danilov Yu.A., Group properties of the Maxwell and Navier-Stokes equations, preprint, Acad. Sci. USSR, Kurchatov In-t of atomic energy, Moscow, 1967.

[3] Fushchych W.I., Symmetry in the problems of mathematical physics, in Theoretic-algebraic investigations in mathematical physics, 6-28, Institute of mathematics, Kiev, 1981.

[4] Fushchych and Popowych R.O., Symmetry reduction and exact solution of the Navier-Stokes equations, Journal of nonlinear mathematical physics, 1994, V. 1, N 1, 2, 75-113, 158-188.

[5] Fushchych and Popowych R.O., Symmetry reduction of the Navier-Stokes equations to linear twodimensional systems of equations, Dopovidi Acad. Sci. Ukrainy, 1992, N 8, 29-37.

[6] Fushchych W.I. and Popowych R.O., Symmetry reduction and exact solution of the Navier-Stokes equations, preprint 93.7, Acad.Sci.Ukraine. Institute of Mathematics, Kiev, 1993.

[7] Fushchych W.I., Shtelen W.M., and Popowych R.O., On reduction of the Navier-Stokes equations to linear heat equations, Dopovidi Acad. Sci. Ukrainy, 1992, N 2, 23-30.

[8] Fushchych W.I., Shtelen W.M., Serov M.I., and Popowych R.O., Q-conditional symmetry of the linear heat equation, Dopovidi Acad. Sci. Ukrainy, 1992, N 12, 29-37.

[9] Kapitanskiy L.V., Group analysis of the Navier-Stokes and Euler equations in the presence of rotational symmetry and new exact solutions of these equations, Dokl. Acad. Sci. USSR, 1978, V.243, N 4, 901-904.

[10] Kapitanskiy L.V., Group analysis of the Navier-Stokes equations in the presence of rotational symmetry and somenew exact solutions, Zapiski nauchnogo sem. LOMI, 1979, V.84, 89-107.

[11] Katkov V.L., Exact solutions of some problems of convection, Prikl. Matem. and Mechan., 1968, V.32, N 3, 482-486.

[12] Popowych R.O., Symmetry reduction and exact solution of the Navier-Stokes equations, PhD thesis, Acad.Sci.Ukraine, Institute of Mathematics, Kiev, 1992.

[13] Popowych R.O., On symmetry and exact solutions of a certain translational equation, Ukr. matem. zhurnal, 1995, V. 47, N 1, 121-125

[14] Pukhnachev V.V., Group properties of the equations of Navier-Stokes in the planar case, PMTF J. Appl. Mech. Tech. Phys., 1960, N 1, 83-90. 